\title{
Physical impairment after acute lung injury
}

\author{
Kenneth Nugent MD
}

The National Institutes of Health NHLBI ARDS network has published more than 150 articles on patients with acute respiratory distress syndrome. The studies have provided essential information about the management of patients with acute respiratory failure requiring mechanical ventilation, including the low tidal volume strategy, fluid and catheter management, and late corticosteroid rescue treatment. This network recently published the ARDSNet Long-Term Outcomes Study (ALTOS) in the American Journal of Respiratory and Critical Care Medicine in May $2014 .{ }^{1}$ This was a prospective longitudinal study of 203 patients who had acute lung injury and were evaluated at 6 and 12 months for physical impairment. The outcome measurements included muscle strength based on the abbreviated Medical Research Council (MRC) score, the 6-minute walk test, and the Medical Outcomes Study SF-36 Physical Function domain score. The MRC score requires testing 12 motor functions, including shoulder abduction, elbow flexion, wrist extension, hip flexion, knee extension, and ankle dorsiflexion. Scores range from 0 (no contraction) to 5 (normal power) resulting in a total score of 0 to 60 . The SF-36 Physical Function domain score is based on answers to ten questions about activities during a typical day with three possible responses (yes-limited a lot, yes-limited a little, no-not limited at all). The mean age in this cohort was $48 \pm 15$ years, $51 \%$ were women, and $92 \%$ lived at home before hospitalization. Eighty percent of the patients had a $\mathrm{PaO}_{2} / \mathrm{FiO}_{2}$ less than 200 at some point during the first three days of mechanical ventilation, $43 \%$ received corticosteroids, $27 \%$ received a neuromuscular blocking agent, and $16 \%$ required dialysis. At the six month followup the percentage of maximum MRC sum score was $92 \% \pm 8 \%$, and $8 \%$ were classified as having ICUacquired weakness with a score of less than 48 out of 60 . The mean percent predicted 6-minute walk test result was $64 \% \pm 22 \%$, and the mean percent predicted SF-36 PF score was $61 \% \pm 36 \%$. There was not much improvement between 6 and 12 months follow-up. Multivariable analysis indicated that corticosteroids and length of time in this ICU adversely influenced these outcomes. There was a strong interaction between these two factors, and patients with longer ICU stays on corticosteroids up to $40 \mathrm{mg}$ per day had worse outcomes. APACHE 3 scores and the use of any neuromuscular blocking agent did not appear to affect physical outcomes. Comorbidity did not have a consistent effect on outcomes.

This study suggests that medical management in the ICU influences physical impairment in patients with acute lung injury. Physical therapy and early mobilization can potentially reduce ICU length of stay and limit the development of muscle weakness and atrophy. Limiting the use of corticosteroids can also reduce adverse effects on muscle strength without any change in mortality. ${ }^{2}$ This study demonstrated that individual muscle testing using the MRC score does not necessarily provide a good indicator of overall impairment since the 6-minute walk test results were clearly worse than individual muscle strength testing scores. In addition, other outcomes were not reported. For example, it would be important to know what percentage of patients returned to their former employment and what percentage of patients has some significant impairment in specific activities of daily living. ${ }^{3}$ Herridge has reported that approximately $77 \%$ of patients who survive hospitalization for acute respiratory failure return to work following hospitalization. ${ }^{4}$ This result suggests that physical performance tests may not correlate with global outcomes such as productive employment. In addition, the ALTOS investigators did not provide information about the use of pulmonary rehabilitation following hospitalization, and we don't know if this contributed to outcomes.

In summary, the ALTOS study suggests that more attention to routine aspects of care, including 
limiting sedation, increasing physical activity in the ICU, and limiting or avoiding corticosteroids, can have important effects on outcomes, including physical impairment and disability. In addition, these patients likely benefit from outpatient rehabilitation. A 2011 Cochrane review has concluded that pulmonary rehabilitation is highly effective and safe in patients with chronic obstructive pulmonary disease who have recently had an acute exacerbation, and we need more studies with rehabilitation in patients with ARDS. ${ }^{5} \mathrm{Fi}-$ nally, patients with ARDS need regular follow-up for complications, such as tracheal stenosis, depression, and post-traumatic stress disorder, post-hospitalization.

Corresponding Author : Kenneth Nugent MD

Author Contact Information: Kenneth.nugent@ttuhsc.edu

DOI: $10.12746 /$ swrccc2014.0208.095

\section{REFERENCES}

1. Needham DM, Wozniak AW, Hough CL, et al. Risk factors for physical impairment after acute lung injury in a national, multicenter study. Am J Respir Crit Care Med 2014; 189:1214-1224. 2. Steinberg KP, Hudson LD, Goodman RB, et al. Efficacy and safety of corticosteroids for persistent acute respiratory distress syndrome. N Engl J Med 2006; 354: 1671-84.

3. Herridge MS, Batt J, Santon CD. ICU-acquired weakness, morbidity, and death. Am J Resp Crit Care Med 2014; 190:360362.

4. Herridge MS, Tansey CM, Matte A, et al. Functional disability 5 years after acute respiratory distress syndrome. $N$ Engl $J$ Med 2011; 364:1293-304.

5. Puhan MA, Gimeno-Santos E, Scharplatz M, et al. Pulmonary rehabilitation following exacerbation of chronic obstructive pulmonary disease. Cochrane Database Syst Rev 2011 Oct 5; (10):

CD005305. doi:10.1002/14651858.CD005305.pub3. 